\title{
Wählerstromanalyse der Nationalratswahl 1999
}

\author{
Erich Neuwirth \\ Institut für Statistik, Operations Research und Computerverfahren \\ Universität Wien
}

\begin{abstract}
Zusammenfassung: Die vorliegende statistische Wählerstromanalyse unterscheidet sich merkbar von der im ORF noch am Wahltag publizierten Analyse. Unterschiede sind insbesondere bei politisch relevanten Fragestellungen wie etwa der Größe des Wählerstroms zwischen der SPÖ und der ÖVP einerseits und der FPÖ andererseits festzustellen. Unsere Analyse wurde im Gegensatz zur ORF-Analyse von einem wissenschaftlich ausgewiesenen Statistiker erstellt. Zumindest aus wissenschaftlich-methodischer Sicht erscheint es daher sinnvoll, die im ORF publizierte Analyse nicht als alleinige Grundlage der Interpretation des Wahlergebnisses heranzuziehen.
\end{abstract}

\section{Zusammenfassung der wichtigsten Analyseergebnisse}

- Die mit Abstand größte Wählerbewegung war ein direkter Übergang von der SPÖ zur FPÖ im Umfang von mehr als 200.000 Stimmen. Diese direkten Verluste waren anteilsmäßig in Wien am höchsten.

- Die zweitstärkste Wählerbewegung war die Wahlenthaltung von 125.000 früheren FPÖ-Wählern, die diesmal nicht gewählt haben.

- Die ÖVP hat ebenfalls ihre stärksten Verluste an die FPÖ erlitten, dieser Wählerstrom ist aber im Umfang wesentlich geringer als die beiden stärksten Ströme. Überdurchschnittlich hoch waren diese Verluste in der Steiermark.

- Das Liberale Forum hat etwa ein Drittel seiner Wähler als Nichtwähler und etwa ein Viertel seiner Wähler an die Grünen verloren.

- Die Grünen konnten ihre stärksten Gewinne auf Kosten des Liberalen Forums erzielen.

\section{Graphische Darstellung der Wählerströme}

Wie ist Abbildung 1 zu lesen? Erläuterung anhand eines Balkens:

Der erste Balken dieser Graphik repräsentiert die SPÖ-Stimmen von 1995. Die Unterteilung dieses Balkens gibt an, wie sich die SPÖ-Wähler von 1995 bei der Wahl 1999 auf sämtliche kandidierenden Parteien verteilt haben. Da die SPÖ über ca. $80 \%$ ihrer Wähler halten konnte, ist der schwarze Teilbalken der größte Teilbalken des gesamten SPÖ-Balkens. Da sie am stärksten an die FPÖ verloren hat, ist der dritte Teilbalken der zweitgrößte Teilbalken.

Der erste Balken in Abbildung 2 stellt die Zusammensetzung der SPÖ-Stimmen von 1999 dar. Man sieht ganz deutlich, daß die SPÖ fast nur von Wählern gewählt wurde, die 


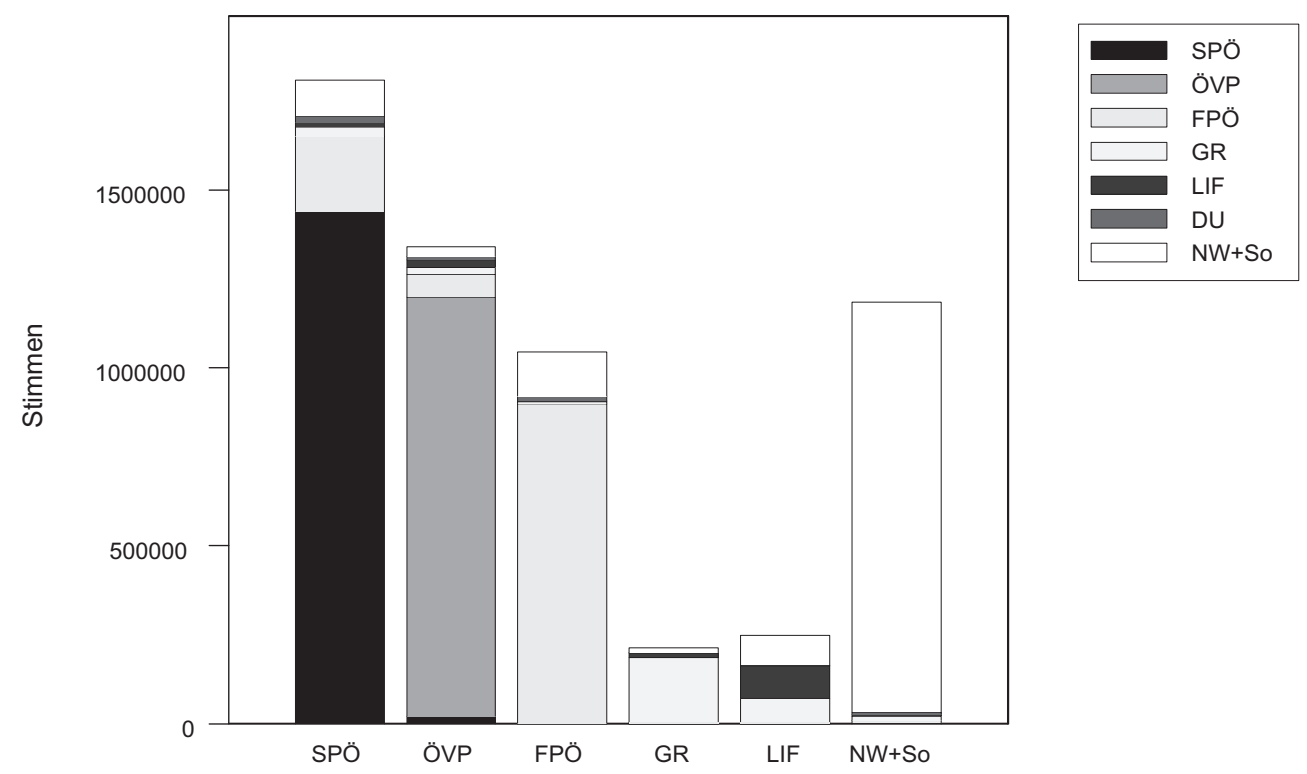

Abbildung 1: Verteilung der Stimmen

sie auch schon bei der NRW 95 gewählt haben. Dazugekommen ist nur eine ganz kleine Gruppe früherer ÖVP-Wähler.

\section{Tabellenteil}

\subsection{Wählerbewegungen in 1000 Stimmen}

\begin{tabular}{|l|r|r|r|r|r|r|r|c|}
\hline & SPÖ & ÖVP & FPÖ & GR & LIF & DU & NW+So & $\begin{array}{c}\text { Schwankungs- } \\
\text { breite }\end{array}$ \\
\hline SPÖ & 1439 & 0 & 213 & 25 & 14 & 17 & 100 & 26 \\
ÖVP & 22 & 1177 & 65 & 18 & 23 & 8 & 27 & 11 \\
FPÖ & 0 & 1 & 898 & 5 & 2 & 13 & 125 & 28 \\
GR & 0 & 0 & 3 & 183 & 13 & 0 & 15 & 14 \\
LIF & 0 & 0 & 4 & 67 & 92 & 1 & 85 & 22 \\
NW+So & 1 & 0 & 8 & 12 & 6 & 6 & 1152 & 14 \\
\hline
\end{tabular}

Wie ist diese Tabelle zu lesen?

Die erste Zeile (die anderen sind analog) bedeutet, daß von allen Wählern, die 1995 die SPÖ gewählt haben, 1439000 auch 1999 wieder die SPÖ gewählt haben. 213000 SPÖWähler von 1995 sind 1999 zur FPÖ gewechselt usw. Die ausgewiesenen Zahlen sind mit einer statistischen Schwankungsbreite, die für jede Partei getrennt in der letzten Spalte ausgewiesen werden, behaftet. Korrekterweise sollte man daher sagen, daß zwischen 1413000 und 1465000 SPÖ-Wählern von 1995 auch 1999 die SPÖ gewählt haben

Erläuterungen zur Schwankungsbreite finden sich in Abschnitt 4. 


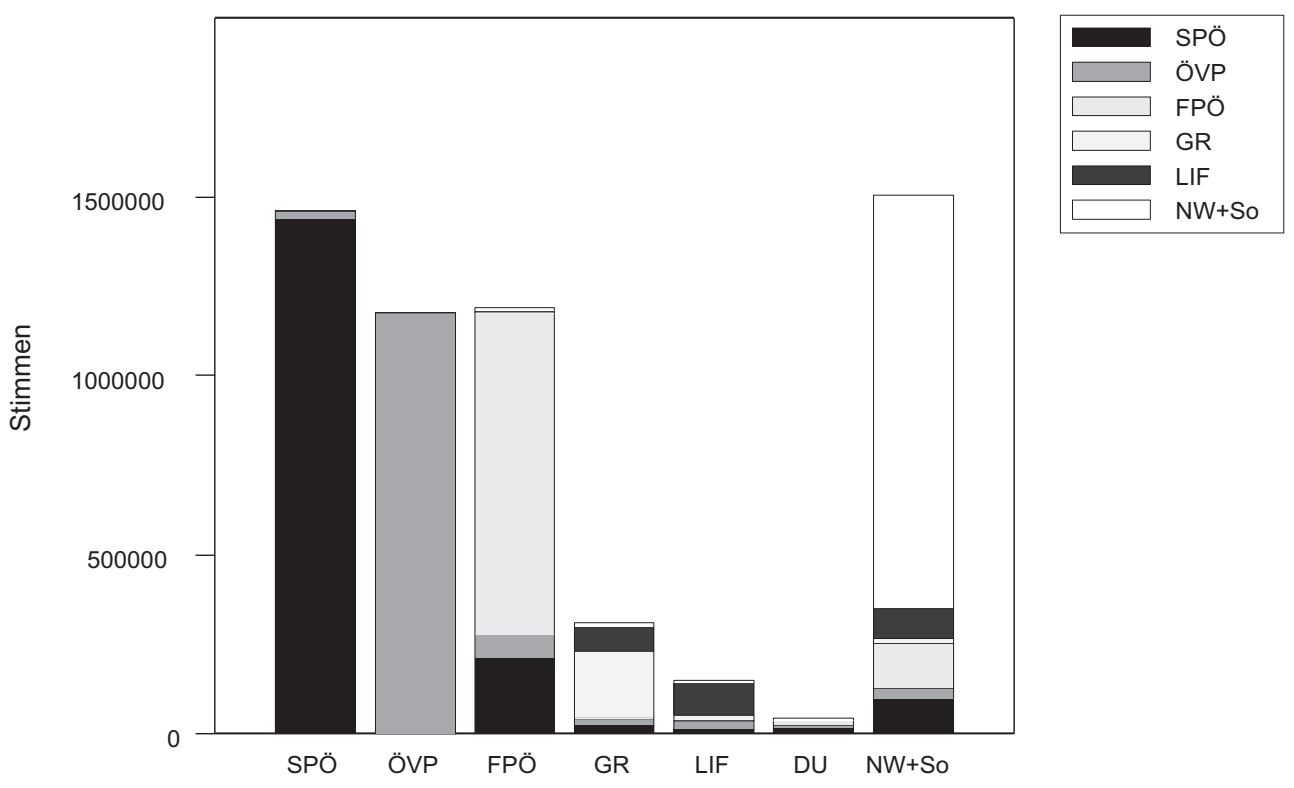

Abbildung 2: Zusammensetzung der Stimmen

\subsection{Saldierte Wählerströme in 1000 Stimmen}

\begin{tabular}{|l|r|r|r|r|r|r|r|}
\hline & SPÖ & ÖVP & FPÖ & GR & LIF & DU & NW+So \\
\hline SPO & 1439 & 22 & -213 & -25 & -14 & -17 & -99 \\
ÖVP & -22 & 1177 & -64 & -18 & -23 & -8 & -27 \\
FPÖ & 213 & 64 & 898 & -2 & 2 & -13 & -117 \\
GR & 25 & 18 & 2 & 183 & 54 & 0 & -3 \\
LIF & 14 & 23 & -2 & -54 & 92 & -1 & -79 \\
DU & 17 & 8 & 13 & 0 & 1 & & 6 \\
NW+So & 99 & 27 & 117 & 3 & 79 & -6 & 0 \\
\hline
\end{tabular}

Die erste Zeile bedeutet, daß die SPÖ etwa 1439000 Wähler von 1995 halten konnte. Die Stimmenbilanz gegenüber der FPÖ beträgt -213000 Stimmen, die SPÖ hat also 213000 Stimmen mehr an die FPÖ verloren als sie von der FPÖ gewonnen hat (da sie, siehe vorige Tabelle, überhaupt keine Stimmen von der FPÖ gewinnen konnte, ist in diesem speziellen Fall Nettobilanz gleich Bruttobilanz).

\subsection{Wählerbewegungen in Prozent der Ausgangspartei}

\begin{tabular}{|l|r|r|r|r|r|r|r|c|}
\hline & SPÖ & ÖVP & FPÖ & GR & LIF & DU & NW+So & $\begin{array}{c}\text { Schwankungs- } \\
\text { breite }\end{array}$ \\
\hline SPÖ & 79.6 & 0.0 & 11.8 & 1.4 & 0.8 & 0.9 & 5.5 & 1.4 \\
ÖVP & 1.6 & 87.9 & 4.8 & 1.3 & 1.7 & 0.6 & 2.0 & 0.8 \\
FPÖ & 0.0 & 0.1 & 86.0 & 0.5 & 0.2 & 1.2 & 12.0 & 2.7 \\
GR & 0.0 & 0.0 & 1.6 & 85.2 & 6.0 & 0.0 & 7.2 & 6.6 \\
LIF & 0.0 & 0.0 & 1.5 & 27.0 & 36.9 & 0.5 & 34.0 & 8.6 \\
NW+So & 0.1 & 0.0 & 0.7 & 1.0 & 0.5 & 0.5 & 97.2 & 1.1 \\
\hline
\end{tabular}


Die erste Zeile gibt an, wie sich die SPÖ-Wähler von 1995 bei der NRW 99 anteilsmäßig auf die Parteien verteilt haben. Die SPÖ konnte 79.6\% ihrer Wähler halten. Sie verlor 11,8\% ihrer Wähler (von 1995) an die FPÖ und 5.5\% ihrer Wähler gaben ihre Stimme nicht für eine der in unserer Untersuchung ausgewiesenen Parteien ab.

\subsection{Zusammensetzung der Parteienstimmen}

\begin{tabular}{|l|r|r|r|r|r|r|}
\hline & SPÖ & ÖVP & FPÖ & GR & LIF & NW+So \\
\hline SPÖ & 98.4 & 1.5 & 0.0 & 0.0 & 0.0 & 0.1 \\
ÖVP & 0.0 & 99.9 & 0.1 & 0.0 & 0.0 & 0.0 \\
FPÖ & 17.9 & 5.4 & 75.3 & 0.3 & 0.3 & 0.7 \\
GR & 8.1 & 5.7 & 1.7 & 59.0 & 21.7 & 3.9 \\
LIF & 9.3 & 15.6 & 1.2 & 8.6 & 61.6 & 3.7 \\
NW & 37.3 & 17.0 & 29.2 & 0.0 & 2.7 & 13.8 \\
\hline
\end{tabular}

98.4\% der SPÖ-Wähler von 1999 haben schon 1995 die SPÖ gewählt, 1.5\% der SPÖWähler haben 1995 die ÖVP gewählt.

\subsection{Wählerbewegungen in Prozent der Wahlberechtigten}

\begin{tabular}{|l|r|r|r|r|r|r|r|c|}
\hline & SPÖ & ÖVP & FPÖ & GR & LIF & DU & NW+So & $\begin{array}{c}\text { Schwankungs- } \\
\text { breite }\end{array}$ \\
\hline SPÖ & 24.6 & 0.0 & 3.7 & 0.4 & 0.2 & 0.3 & 1.7 & 0.4 \\
ÖVP & 0.4 & 20.2 & 1.1 & 0.3 & 0.4 & 0.1 & 0.5 & 0.2 \\
FPÖ & 0.0 & 0.0 & 15.4 & 0.1 & 0.0 & 0.2 & 2.1 & 0.5 \\
GR & 0.0 & 0.0 & 0.1 & 3.1 & 0.2 & 0.0 & 0.3 & 0.2 \\
LIF & 0.0 & 0.0 & 0.1 & 1.2 & 1.6 & 0.0 & 1.4 & 0.4 \\
NW+So & 0.0 & 0.0 & 0.1 & 0.2 & 0.1 & 0.1 & 19.7 & 0.2 \\
\hline
\end{tabular}

26.4\% der Wahlberechtigten haben 1995 und 1999 die SPÖ gewählt, von der SPÖ zur FPÖ gewechselt sind 3.7\% der Wahlberechtigten, und jene SPÖ-Wähler von 1995, die diesmal keine Stimme für eine der 6 in dieser Analyse ausgewiesenen Parteien abgegeben haben, waren dem Umfang nach $1.7 \%$ der Wahlberechtigten.

Wie im folgenden noch dargestellt entstehen alle diese Tabellen durch Konsolidierung von jeweils für die einzelnen Bundesländer durchgeführten Analysen. Daher gibt es die angeführten Tabellen nicht nur bundesweit, sondern auch bundesländerspezifisch. Diese Tabellen würden allerdings den Umfang dieses Artikels übermäßig erweitern und sind daher hier nicht angeführt. Sie stehen jedoch am WWW unter der URL

http://www .smc .univie.ac .at/ n neuwirth/wstrom99/

zur Verfügung.

\section{Methodische Bemerkungen}

Die Datengrundlage unserer Analyse sind die ausgezählten Gemeindeergebnisse aller österreichischen Gemeinden. Die Wahlkartenstimmen können in unserer Analyse nicht 
berücksichtigt werden, weil sie keinen Gemeinden zugeordnet werden können und daher nicht mit Wahlberechtigtenzahlen verbunden werden können.

Die unserer Analyse zugrunde liegenden Modellannahmen und Schätzverfahren sind in Neuwirth (1994) vollständig dokumentiert und in Neuwirth (1984a) und Neuwirth (1984b) in etwas populärerer Form dargestellt.

Grundlage des Modells ist pro Bundesland ein Wählerübergangsmodell, das für alle Wähler, die bei der Vorwahl eine bestimmte Partei gewählt haben, konstante und innerhalb des Bundeslandes räumlich homogene Übergangswahrscheinlichkeiten zu allen bei der neuen Wahl kandidierenden Parteien annimmt. Die Gruppe der Nichtwähler wird dabei wie eine weitere Partei behandelt.

Aus diesen Annahmen folgt, daß das neue Wahlergebnis für alle Gemeinden eines Bundeslandes durch ein lineares Modell mit dem alten Wahlergebnis als Prädiktorvariablen dargestellt werden kann. Das Wählerübergangsmodell, das für den einzelnen Wähler eine Multinomialverteilung annimmt, führt dazu, daß die Gemeindergebnisse als annähernd normalverteilt angenommen werden können. Die Varianzen der Gemeindeergebnisse sind jedoch nicht konstant, sondern abhängig von der Größe der Gemeinden. Somit kann man zur Schätzung der Übergangswahrscheinlichkeiten nicht einfach einen simplen, Varianzhomogenität voraussetzenden Regressionsansatz wählen. Man kann aber die Maximum-Likelihood-Gleichungen im Modell explizit aufschreiben und mit numerischen Optimierungsverfahren auch lösen. Zu berücksichtigen ist auch, daß die Übergangswahrscheinlichkeiten natürlich nur Werte zwischen 0 und 1 annehmen dürfen. Daher ist die zu lösende Maximum-Likelihood-Aufgabe eine Optimierungsaufgabe mit linearen Nebenbedingungen. Die Einhaltung dieser Nebenbedingungen kann bei simpler Anwendung von Formeln zur Berechnung von Regressionskoeffizienten nicht garantiert werden, und in so einem simplifizierenden Regressionsansatz wird daher in manchen Fällen von Nichtstatistikern "von Hand aus" nachgebessert. Der üblichen statistischen Argumentation folgend wissen wir, daß der Maximum-Likelihood-Schätzer nicht schlechter als der Regressionsschätzer ohne Nebenbedingungen ist. Daher können wir die Varianzen der Schätzer der Übergangskoeffizienten durch die Varianzen der unrestringierten Regressionsschätzer nach oben abschätzen und so können wir auch Konfidenzintervalle für die geschätzten Übergangswahrscheinlichkeiten angeben.

In den Tabellen, wo diese Konfidenzintervalle jeweils für alle in einer Zeile erscheinenden Schätzwerte konstant sind, weist die mit Schwankungsbreite bezeichnete Spalte wie üblich die halbe Konfidenzintervallbreite für ein Konfidenzniveau von 95\% aus. Um die Tabellen nicht zu überfrachten, wurden die entsprechenden Intervallbreiten in den Tabellen, wo sie für verschiedene Werte in einer Zeile schon verschieden sind, nicht explizit angeführt. Sie sind aber natürlich jederzeit ganz einfach errechenbar.

Wegen der geringen Fallzahl für das statistische Modell für Wien (es stehen nur die 23 Bezirke, aber keine Sprengelergebnisse zur Verfügung) sind die Schwankungsbreiten in Wien besonders groß. Man sollte also politische Schlußfolgerungen über die Größe mancher Wählerströme in Wien sehr behutsam interpretieren. 


\section{Literatur}

Erich Neuwirth. Schätzung von Wählerübergangswahrscheinlichkeiten. In Manfred Holler, editor, Wahlanalyse, tuduv-Verlag, München, 1984a.

Erich Neuwirth. Lineare Modelle, Wahlanalyse und Wählerstromrechnung. Mitteilungsblatt der Österreichischen Gesellschaft für Statistik und Informatik, 53, 1984b.

Erich Neuwirth. Prognoserechnung am Beispiel der Wahlhochrechnung. In P. Mertens, Prognoserechnung, Physica-Verlag, Heidelberg, 1994.

Adresse des Autors:

Ao.Univ.-Prof. Dr. Erich Neuwirth

Institut für Statistik, Operations Research und Computerverfahren

Universität Wien

Universitätsstr. 5

A-1010 Wien

Tel. +431 4277 / 38624

Fax +431 4277 / 9386

E-Mail: Erich.Neuwirth@univie.ac.at 